\title{
Clinical characteristics and laboratory analyses of acute myeloid leukemia with $\mathrm{t}(16 ; 21)(\mathbf{p 1 1} ; \mathbf{q} 22)$
}

\author{
ZHIFEN ZHANG* ${ }^{*}$, JIANWEN ZOU*, YUANTANG LI, ZHANFENG LIU, RUI XU, WENJUN TIAN, \\ ZONGCHEN ZHAO, HUI SUN, JINGYING HAN, JIA WANG, BINGCHANG ZHANG and YING JU
}

\author{
Department of Clinical Laboratory, Shandong Provincial Hospital Affiliated to Shandong University, \\ Jinan, Shandong 250021, P.R. China
}

Received May 11, 2014; Accepted February 5, 2015

DOI: $10.3892 / 01.2015 .3051$

\begin{abstract}
The present study reviewed three patients with acute myeloid leukemia (AML) who had the specific genetic abnormality $\mathrm{t}(16 ; 21)(\mathrm{p} 11 ; \mathrm{q} 22)$. To investigate the clinical and laboratory characteristics of AML with $\mathrm{t}(16 ; 21)(\mathrm{p} 11 ; \mathrm{q} 22)$ translocation, the similarities and differences of clinical characteristics and laboratory examinations were compared, and a literature review was conducted. According to the French-American-British classification system, patient 1 was M4, patient 2 was M1 and patient 3 was M2. The cytogenetic aberrations were $46, \mathrm{XY}, \mathrm{t}(16 ; 21)$ (p11;q22)/47, idem, +21 for patient 1 and 46, XX,t(16;21)(p11;q22) for patients 2 and 3. Cytophagocytosis and cluster of differentiation 56 antigen expression were found in all three cases. The prognosis was poor in all the cases. AML with $\mathrm{t}(16 ; 21)(\mathrm{p} 11 ; \mathrm{q} 22)$ is a specific subtype of AML that exhibits unique characteristics of morphology, immunology, cytogenetics and clinical features, as well as a poor prognosis. Stem cell transplantation may be the first and only choice for treatment.
\end{abstract}

\section{Introduction}

Acute myeloid leukemia (AML) is a type of hematologic malignancy. The peak age of onset is $>60$ years old (1). AML is frequently accompanied by specific cytogenetic aberrations. $\mathrm{t}(16 ; 21)$ (p11;q22) is a rare and non-random chromosomal translocation that causes the rearrangement of erythroblast transformation specific-related gene (ERG) on chromosome 21 and translocated in sarcoma/fused in sarcoma (TLS/FUS) on chromosome 16, forming the TLS/FUS-ERG fusion gene. $\mathrm{t}(16 ; 21)$ (p11;q22) occurs with an incidence of $1 \%$ in AML (2). The morphology, immunology and clinical manifestation of

Correspondence to: Dr Ying Ju, Department of Clinical Laboratory, Shandong Provincial Hospital Affiliated to Shandong University, 324 Jinwu Weiqi Road, Jinan, Shandong 250021, P.R. China

E-mail: juying2003@126.com

*Contributed equally

Key words: translocation, cytophagocytosis cytogenetics, acute leukemia this translocation are distinct from other subtypes of AML. Literature associated with $\mathrm{t}(16 ; 21)(\mathrm{p} 11 ; \mathrm{q} 22)$ AML is scarce. The present study reports three patients with AML and $t(16 ; 21)$ (p11;q22) who exhibited unique characteristics compared with other subtypes of AML. Written infomred consent was obtained from the family of the patient.

\section{Case report}

Patient 1. Patient 1 was a 17-year-old male. Three weeks prior to presentation, the patient exhibited a fever of unknown origin with a peak temperature of $38.5^{\circ} \mathrm{C}$. The patient did not exhibit chest tightness, chest pain or a cough. He was administered antibiotics and dexamethasone in a local hospital, and his body temperature returned to normal. The patient was then transferred to the Shandong Provincial Hospital (Jinan, China) for further diagnosis and treatment. Peripheral blood count results showed a hemoglobin level of $77 \mathrm{~g} / 1$, a platelet (PLT) count of $14 \times 10^{9} / 1$, and a white blood cell (WBC) count of $46.82 \times 10^{9} / 1$ (10.5\% segmented neutrophils, $12 \%$ lymphocytes and $77.5 \%$ leukemic blast cells). A physical examination revealed an anemic appearance, no skin or mucosal bleeding, no pain at the bottom of the sternum, normal heart and lung exams and no hepatosplenomegaly.

Bone marrow aspirate was stained by Wright-Giemsa stain (Sigma-Aldrich, St. Louis, MO, USA), and showed a hypercellular marrow with $77.5 \%$ leukemic blast cells. Hemophagocytosis and vacuolation were observed in the leukemic cells (Fig. 1). Phagocytosed blood cells included WBCs, red blood cells and PLTs. Cytochemical staining of leukemic blast cells included peroxidase, naphthol AS-D chloroacetate esterase and periodic acid-schiff reaction staining (all obtained from Zhuhai Baso Biological Technology Co., Ltd., Zhuhai, China). Morphology and cytochemical staining of leukemic cells are shown in Table I. Blasts were detected using flow cytometry (BD Biosciences, San Jose, CA, USA) for the antigens shown in Table II, including cluster of differentiation (CD)117, CD13, CD34, CD56, CD38, CD33, CD15, myeloperoxidase (MPO) and human leukocyte antigen-DR (HLA-DR) (all purchased from BD Biosciences). Antigens CD19, CD10, CD20, CD7, CD11b, CD64, CD3 and CD138 were all negative (data not shown). Under the French-American-British (FAB) classification system, patient 1 was AML-M4. Bone marrow cells were incubated with RPMI 1640 medium (Biosource International, Inc., Camarillo, 
CA, USA) containing $10 \%$ heat-inactivated fetal bovine serum for $24 \mathrm{~h}$ and chromosomes were analyzed using the Wright-Giemsa stain. VideoTesT-Karyo3.1 Chromosome Analysis system (Video TesT, Petersburg, Russia) was used to analyze the karyogram. The karyotypes were described according to the International System for Human Cytogenetic Nomenclature 2009 (3). Patient 1 was 46,XY,t(16;21)(p11;q22)(16)/47,idem,+21(4) (Fig. 2A). Bio-Rad CFX96 fluorescent quantitative polymerase chain reaction (PCR) system (Bio-Rad Laboratories, Hercules, CA, USA) and reverse-transcription PCR kit [Tiangen Biotech (Beijing) Co., Ltd., Beijing, China] were used for detection. The patient was negative for the following fusion genes: AML1-Eight-Twenty-One, promyelocytic leukemia-retinoic acid receptor $\alpha$ (long, short, variant), core binding factor $\beta$-myosin $11 \mathrm{~A}$, E2A-pre-B-cell leukemia homeobox 1, breakpoint cluster region-Abelson murine leukemia viral oncogene homolog 1 (P210, P190), translocation ets-like gene-AML1, SCL interrupting locus-transcription-activator-like 1 , and mixed lineage leukemia-AF4. The TLS/FUS-ERG fusion gene was positive.

Patient 1 was treated with DA (administration of $40 \mathrm{mg}$ daunorubicin once a day between days 1 and 3 and $150 \mathrm{mg}$ cytarabine once a day between days 1 and 7) for the first treatment cycle. The next two treatment cycles were administration of HA ( $3 \mathrm{mg}$ homoharringtonine once a day between days 1 and 3 and $150 \mathrm{mg}$ cytarabine once a day between days 1 and 7). The fourth treatment cycle was administration of $30 \mathrm{mg}$ pirarubicin once a day between days 1 and 3 and $150 \mathrm{mg}$ cytarabine once a day between days 1 and 7. The fifth and sixth treatment cycles were HA, subsequent to which the patient achieved complete remission. The seventh treatment cycle was administration of $100 \mathrm{mg}$ etoposide VP16 once a day between days 1 and 5 and $150 \mathrm{mg}$ cytarabine once a day between days 1 and 7. The eighth treatment cycle was medium cytarabine (administration of $1.0 \mathrm{~g}$ twice a day between days 1 and 7). The ninth treatment cycle occurred following admission to the hospital. Bone marrow aspirate showed $30.5 \%$ leukemic blast cells. The patient was considered to exhibit a recurrence and was treated with administration of $10 \mathrm{mg}$ idarubicin hydrochloride once a day between days 1 and 3 and $150 \mathrm{mg}$ cytarabine once a day between days 1 and 5). The patient received nine courses of chemotherapy and did not achieve complete molecular and hematological remission. The patient succumbed after 2 years.

Patient 2. Patient 2 was a 10-year-old female who presented with a recurrent fever that lasted $>2$ weeks and leg pain that lasted 10 days. Two weeks previously, the patient appeared pale and exhibited a fever and a non-productive cough with no evident cause. She did not exhibit nausea, vomiting, abdominal pain or diarrhea. The fever was resolved following drug treatment at a local clinic. A physical examination revealed an anemic appearance, diffuse petechiae and no superficial lymph node enlargement. Peripheral blood count results included a hemoglobin level of $40 \mathrm{~g} / 1$, a PLT count of $6 \times 10^{9} / 1$, and a WBC count of $55.59 \times 10^{9} / 1$ [2.5\% segmented neutrophils, $4 \%$ lymphocytes and $93.5 \%$ leukemic blast cells (shown in the hypercellular bone marrow aspirate)]. The cytochemical staining and immunological analysis were consistent with AML. The FAB classification M1 was established. Morphology and cytochemical staining of leukemic cells are shown in Table I. Hemophagocytosis and vacuolation were observed in the leukemic cells
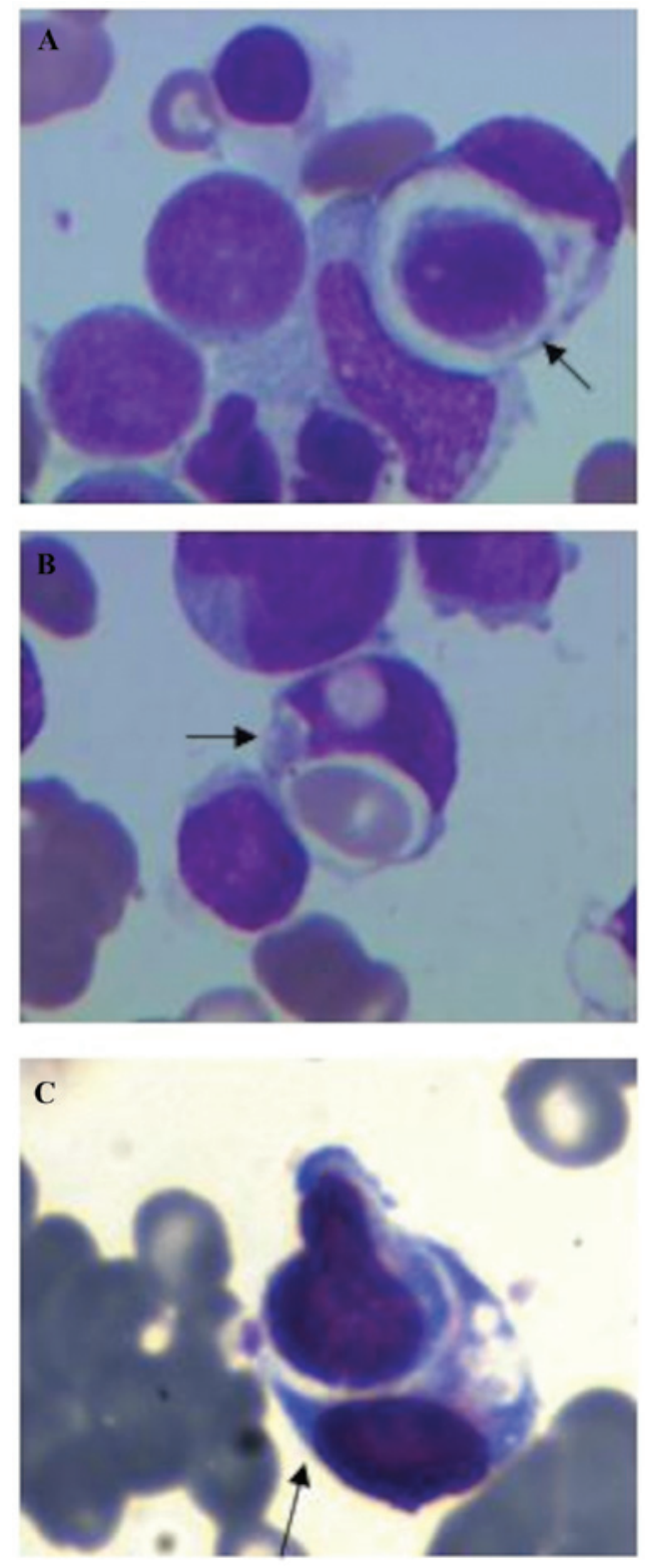

Figure 1. Phagocytosis of leukemic cells. Blasts observed in the bone marrow aspirate phagocytosed (A) white blood cells in patient 1 , (B) red blood cells in patient 2 and $(C)$ white blood cells in patient 3. Wright-Giemsa staining; original magnification, $\mathrm{x} 1,000$.

(Fig. 1). Blast antigens are shown in Table II. The karyotype was 46,XX,t(16;21)(p11;q22)(20) (Fig. 2B). The TLS/FUS-ERG fusion gene was positive and the other genes were all negative. The patient received nine days of daunorubicin and etoposide chemotherapy and was in a critical condition from an infection, resulting in mortality after 1 month.

Patient 3. Patient 3 was a 54-year-old female who had a history of epilepsy of $>20$ years and was on scheduled antiepileptic medications. At 15 days prior to presentation, the patient ceased the use of the antiepileptic medications due to a sustained severe headache. The patient then exhibited weakness, and leg ecchymoses were found. Upon physical examination, the patient was revealed to have clear mental status, normal development, pallor, ecchymoses in the two lower limbs and no superficial lymph node enlargement. Peripheral blood count 
Table I. Morphology and cytochemical staining of the leukemic blast cells of the three patients at diagnosis.

\begin{tabular}{lccccccc}
\hline Bone marrow & $\begin{array}{c}\text { Leukemic } \\
\text { hyperplasia } \\
\text { degree }\end{array}$ & $\begin{array}{c}\text { Auer rods } \\
\text { cells }(\%)\end{array}$ & or bodies & Cytophagocytosis & POX $^{\text {a }}$ & AS-DCE $^{\text {a }}$ & PAS $^{\text {a }}$ \\
\hline 1 & Active & 77.5 & Not observed & Yes & Positive & Negative & Negative \\
2 & Active & 93.5 & Not observed & Yes & Positive & Negative & Partial positive \\
3 & Active & 62.0 & Not observed & Yes & Positive & Negative & Positive \\
\hline
\end{tabular}

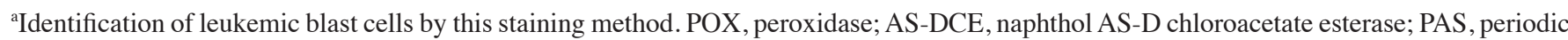
acid-schiff reaction.

Table II. Percentage of blasts with antigen positive cells.

\begin{tabular}{lccccccccc}
\hline Patient & $\mathrm{CD} 117(\%)$ & $\mathrm{CD} 13(\%)$ & $\mathrm{CD} 34(\%)$ & $\mathrm{CD} 56(\%)$ & $\mathrm{CD} 38(\%)$ & $\mathrm{CD} 33(\%)$ & $\mathrm{CD} 15(\%)$ & $\mathrm{MPO}(\%)$ & HLA-DR (\%) \\
\hline 1 & 85.49 & 65.95 & 89.80 & 79.30 & 26.83 & - & - & 79.69 & 62.91 \\
2 & 66.68 & 98.67 & 99.50 & 93.41 & 38.45 & 99.65 & 48.58 & - & - \\
3 & 61.75 & 52.11 & 91.97 & 71.60 & 75.40 & 94.18 & 55.54 & - & 39.50 \\
\hline
\end{tabular}

-, negative; CD, cluster of differentiation; MPO, myeloperoxidase; HLA-DR, human leukocyte antigen-DR.

results included a hemoglobin level of $86 \mathrm{~g} / 1$, PLT count of $46 \times 10^{9} / 1$, and WBC count of $2.0 \times 10^{9} / 1$ [18\% segmented neutrophils, $13 \%$ lymphocytes, $7 \%$ monocytes and $62 \%$ leukemic blast cells (shown in the hypercellular bone marrow aspirate)]. The cytochemical staining and immunological analysis were consistent with AML. FAB classification M2 was established. Morphology and cytochemical staining of leukemic cells are shown in Table I. Hemophagocytosis and vacuolation were observed in the leukemic cells (Fig. 1). Blast antigens are shown in Table II. The karyotype was 46,XX,t(16;21)(p11;q22) (20) (Fig. 2C). The TLS/FUS-ERG fusion gene was positive and the other genes were all negative. The patient was treated with DA and cytarabine plus mitoxantrone, and subsequently received three courses of chemotherapy, without attaining remission. The patient survived for two years following the initial diagnosis

\section{Discussion}

$\mathrm{t}(16 ; 21)(\mathrm{p} 11 ; \mathrm{q} 22)$ is a unique subtype of AML. For three patients reported previously, the age at diagnosis had a range of 1-81 years, with a median age of 26 years (1), and had a poor prognosis. $\mathrm{t}(16 ; 21)(\mathrm{p} 11 ; \mathrm{q} 22)$ results in the chimeric transcript TLS/FUS-ERG (4). This translocation causes the replacement of the RNA-binding domain of FUS with the DNA-binding domain of ERG $(5,6)$. This fusion gene is thought to be responsible for the leukemogenesis of AML harboring $\mathrm{t}(16 ; 21)(7)$. Four transcripts found in $\mathrm{AML}$ with $\mathrm{t}(16 ; 21)$ have been designated as types $\mathrm{A}, \mathrm{B}, \mathrm{C}$ and $\mathrm{D}$, corresponding to the chimeric products of $255,211,179$ and 349 bp, respectively. The $t(16 ; 21)$ translocation has been reported in different types of leukemia $(5,7)$. FAB classifications M3, M2 and M5 are the most common, and M1 and M4 are the next most common. These classifications included chronic myeloid leukemia, acute lymphoblastic leukemia and myelodysplastic syndrome (7-10). This result indicates that $\mathrm{t}(16 ; 21)(\mathrm{p} 11 ; \mathrm{q} 22)$ and the TLS/FUS-ERG chimeric transcript do not occur exclusively in AML (11).

Of the published karyotypes of AML with the TLS/FUS-ERG chimeric transcript, some were $\mathrm{t}(16 ; 21)(\mathrm{pll} ; \mathrm{q} 22)$, while others had additional abnormities, including $+10,+12,+8,+6,-9$, ins $(7 ; 2), \operatorname{del}(9)(q 13 ; q 33)$, $+\operatorname{der}(21)$ and $\operatorname{del}(15)(q 11 ; q 15)(1)$. In the present report, the karyotype of patient 1 had +21 as an additional abnormality. This combination has not been reported in previous studies. The small chromosome 21, harboring 300 genes, may be involved in numerous structural aberrations, including translocations, deletions and amplifications in leukemia and lymphoma. Genes located on chromosome 21 have been identified to play important roles in tumorigenesis (12). Trisomy 21 is the most common cytogenetic abnormality at birth and one of the most recurrent aneuploidies in leukemia (13). Constitutional +21 of Down's syndrome is associated with increased risk for childhood leukemia (14). The elevated incidence of acute megakaryocytic leukemia in young children with +21 is estimated at $\sim 500$-fold $(13,15)$.

Immunophenotyping results have been inconsistent for AML with $\mathrm{t}(16 ; 21)$. Marosi et al (16) found that the leukemic cells expressed CD7, CD71, CD38, CD15, HLA-DR, CDw65, CD13, CD33 and CD42b (17); however, Nobbs et al (17) reported that the leukemic cells mainly expressed CD34, HLA-DR, CD7, CD13 and CD33, and did not express CD2, CD10, CD19, CD20, CD14 and terminal deoxynucleotidyl transferase. Tan et al (18) reported a 54-year-old man whose leukemic cells mainly expressed CD7, CD13, CD33, CD34, CD117, CD56, CD64, HLA-DR and cytoplasmic (c)MPO, with positive percentages of 89.9, 53.5, 75.8, 96.7, 99.0, 41.4, 19.9, 98.4 and 97.5\%, respectively, while CD11b, CD14, CD15, CD19, CD3, CD45e and CD79a were not expressed (19). In the present three cases, the leukemic blast cells all highly expressed CD7, CD13, CD34, 
A

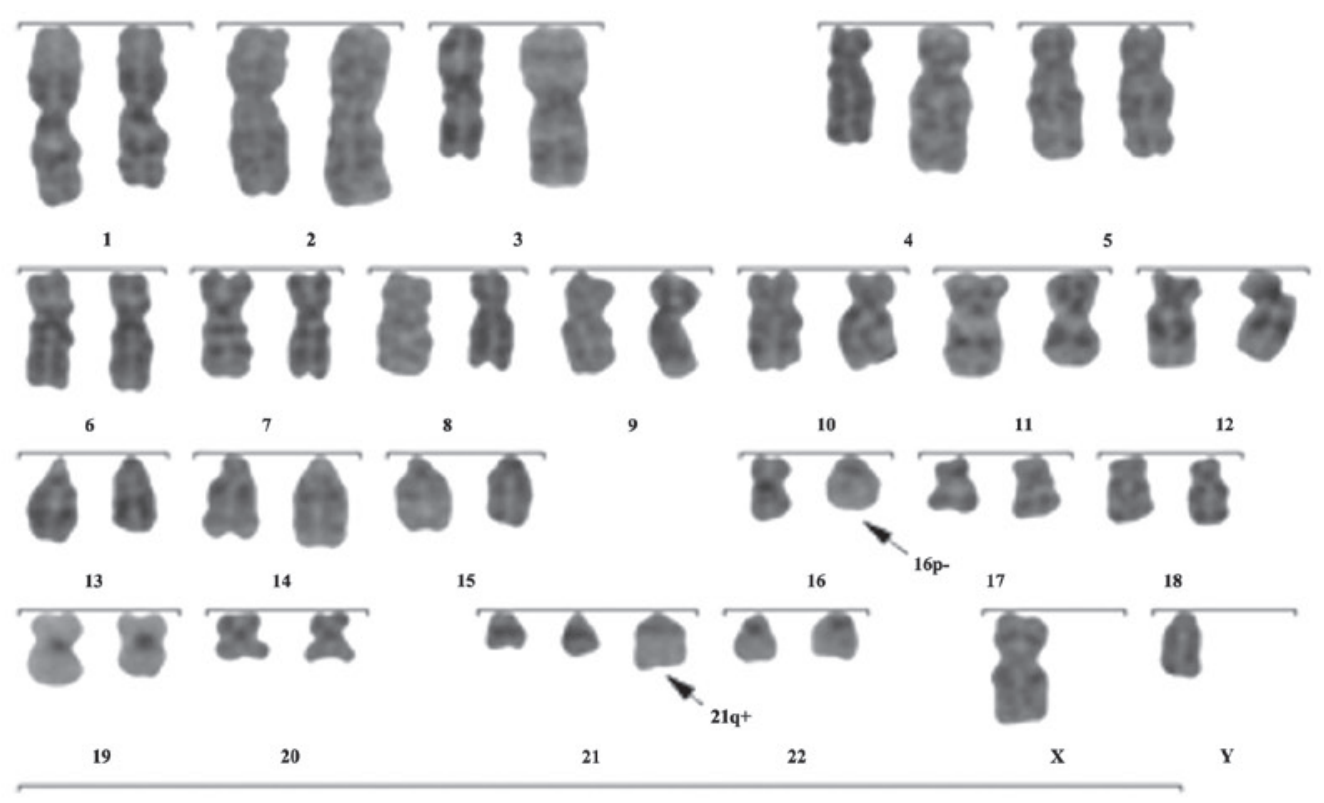

B
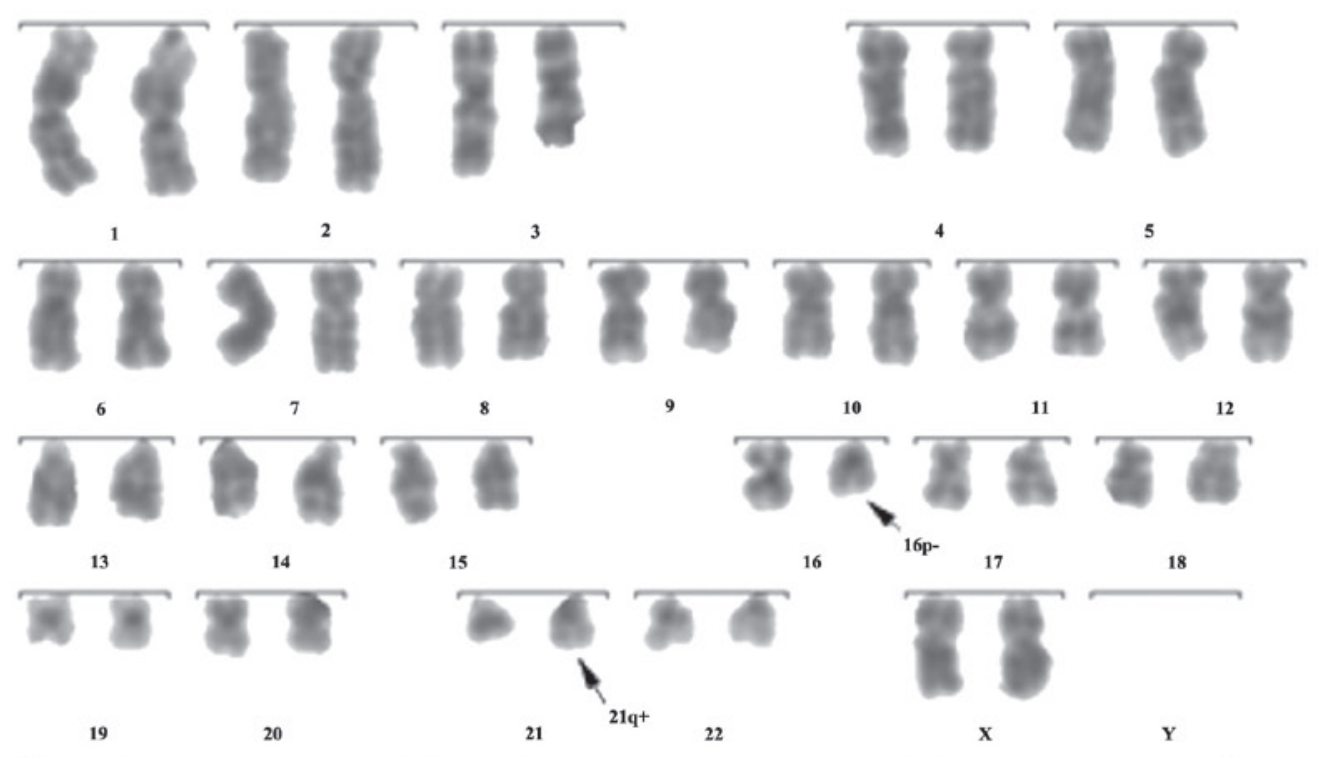

C
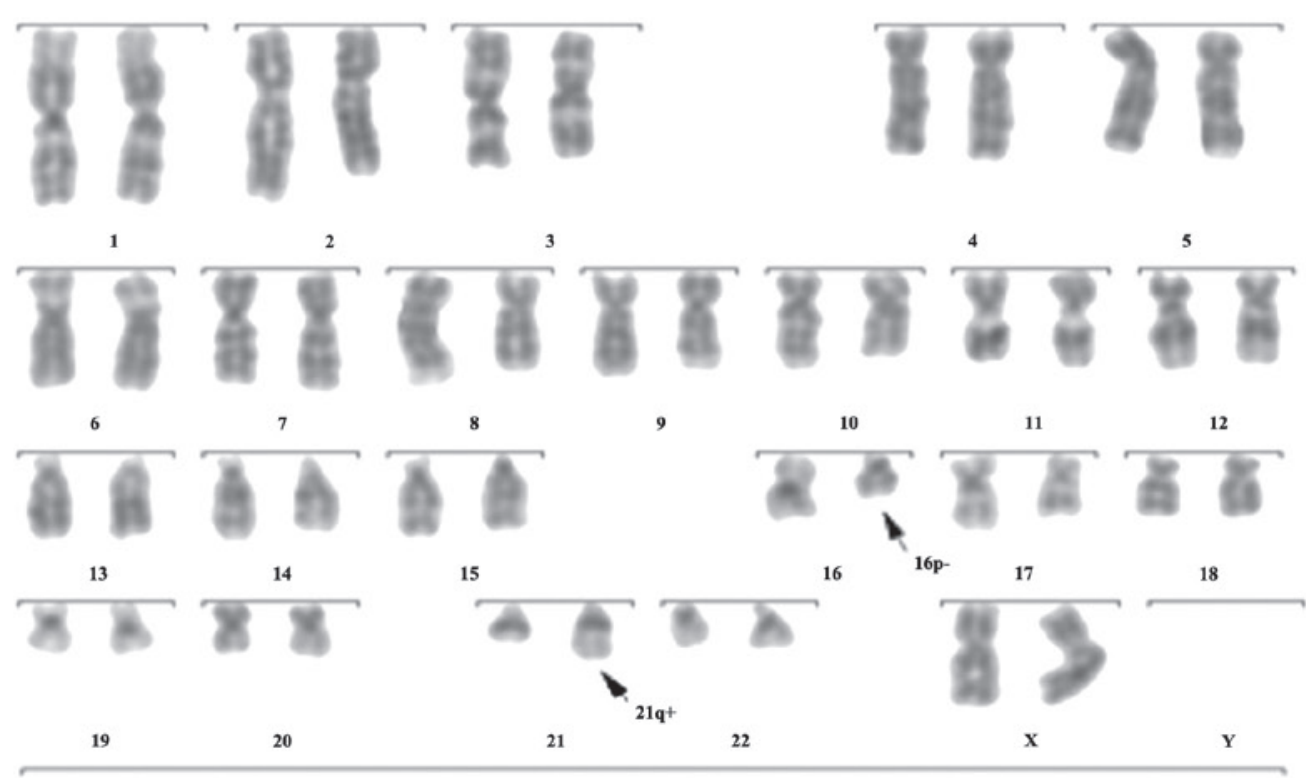

Figure 2. Karyograms of patients using Giemsa banding (stain, Wright-Giemsa). (A) Patient 1: 46,XY,t(16;21)(p11;q22)(16)/47,XY,t(16;21)(p11;q22),+21(4). (B) Patient 2: 46,XX,t(16;21)(p11;q22)(20). (C) Patient 3: 46,XX,t(16;21)(p11;q22)(20). 
CD56 and CD38. Additionally, patient 1 expressed HLA-DR and cMPO; patient 2 expressed CD33 and CD15; and patient 3 expressed CD33, CD15 and HLA-DR. This indicates that the blast cells of AML with $t(16 ; 21)$ originate from an earlier stage of myeloid cell differentiation. Granulocytes, mononuclear cells, megakaryocytes and red blood cells may be involved.

Jekarl et al (2) reported that the average percentage of CD56 positive blasts in AML with $\mathrm{t}(16 ; 21)$ was $45 \%$. Imashuku et al (9) suggested that the morphology of bone marrow aspirate of patients with $A M L$ and $t(16 ; 21)$ was unique. Leukemic cells phagocytose WBCs, red blood cells, PLTs and other leukemic cells. The cytoplasms of leukemic cells have pseudopodia and one or more, occasionally alveolate-like, vacuoles. Increased eosinophilia was observed in the bone marrow or peripheral blood of certain patients, but Auer rods were uncommon. Hemophagocytosis and vacuolation are closely associated with the CD56 expression of blast cells (9). In the present cases, hemophagocytosis and vacuolation of the blast cells were typical, Auer rods were not observed and eosinophilia was absent. These findings were consistent with the reports of Imashuku et al (9), but not with that of Wu et al, who reported that the other five patients in China did not exhibit the aforementioned characteristics (19). Therefore, it has been suggested that heterogeneity exists in the bone marrow aspirate morphology of patients with AML and $\mathrm{t}(16 ; 21)$.

The progression of AML is faster in patients with $t(16 ; 21)(\mathrm{p} 11 ; \mathrm{q} 22)$ than in patients with other subtypes of AML. With conventional chemotherapy, complete remission is difficult to achieve, and the duration of remission is short. Early relapse is common, and the median survival time is 16 months (7). Therefore, $\mathrm{t}(16 ; 21)(\mathrm{p} 11 ; \mathrm{q} 22)$ can be used as an independent marker for poor prognosis (19). In the present case report, Patient 1 succumbed after 2 years, Patient 2 succumbed after 1 month, and Patient 3 did not sustain remission despite three courses of chemotherapy. Therefore, the treatment results were not adequate compared with conventional chemotherapy regimens, and hematopoietic stem cell transplantation should be performed (7).

\section{Acknowledgements}

This study was supported by grants from the National Natural Science Foundation of China (no. 81000731) and the Promotive Research Fund for Excellent Young and Middle-Aged Scientists of Shandong Province (no. BS2010YY045).

\section{References}

1. Kim J, Park TS, Song J, et al: Detection of FUS-ERG chimeric transcript in two cases of acute myeloid leukemia with $t(16 ; 21)$ (p11.2;q22) with unusual characteristics. Cancer Genet Cytogenet 194: 111-118, 2009.

2. Jekarl DW, Kim M, Lim J, et al: CD56 antigen expression and hemophagocytosis of leukemic cells in acute myeloid leukemia with $\mathrm{t}(16 ; 21)$ (p11;q22). Int J Hematol 92: 306-313, 2010.
3. Park TS, Lee ST, Song J, et al: A tandem triplication, trp (1) (q21q32), in a patient with follicular lymphoma: a case study and review of the literature. Cancer Genet Cytogenet 189: 127-131, 2009.

4. Ismael O, Shimada A, Elmahdi S, et al: RUNX1 mutation associated with clonal evolution in relapsed pediatric acute myeloid leukemia with t(16;21) (p11;q22). Int J Hematol 99: 169-174, 2014.

5. Ichikawa H, Shimizu K, Hayashi Y and Ohki M: An RNA-binding protein gene, TLS/FUS, is fused to ERG in human myeloid leukemia with $\mathrm{t}(16 ; 21)$ chromosomal translocation. Cancer Res 54: 2865-2868, 1994.

6. Panagopoulos I, Aman P, Fioretos T, et al: Fusion of the FUS gene with ERG in acute myeloid leukemia with $\mathrm{t}(16 ; 21)$ (p11;q22). Genes Chromosomes Cancer 11: 256-262, 1994.

7. Kong XT, Ida K, Ichikawa H, Shimizu K, et al: Consistent detection of TLS/FUS-ERG chimeric transcripts in acute myeloid leukemia with $\mathrm{t}(16 ; 21)(\mathrm{p} 11 ; \mathrm{q} 22)$ and identification of a novel transcript. Blood 90: 1192-1199, 1997.

8. Oh SH, Park TS, Choi JR, et al: Two childhood cases of acute leukemia with $\mathrm{t}(16 ; 21)$ (p11.2;q22): second case report of infantile acute lymphoblastic leukemia with unusual type of FUS-ERG chimeric transcript. Cancer Genet Cytogenet 200: 180-183, 2010.

9. Imashuku S, Hibi S, Sako M, et al: Hemophagocytosis by leukemic blasts in 7 acute myeloid leukemia cases with $\mathrm{t}(16 ; 21)$ (p11;q22): common morphologic characteristics for this type of leukemia. Cancer 88: 1970-1975, 2000.

10. Kawashima N, Shimada A, Taketani T, et al: Childhood acute myeloid leukemia with bone marrow eosinophilia caused by $\mathrm{t}(16 ; 21)$ (q24;q22). Int J Hematol 95: 577-580, 2012.

11. Huang Z, Chai YH, He HL, Li JQ, Lu J and Shao XJ: The clinical and laboratory study of TLS-ERG fusion gene in seven cases of childrens' acute leukemia. Journal of Leukemia \& Lymphoma 16: 352-354, 2007 (In Chinese).

12. Fonatsch C: The role of chromosome 21 in hematology and oncology. Gene's chromosomes cancer 49: 497-508, 2010.

13. Klusmann JH, Creutzig U, Zimmermann M, et al: Treatment and prognostic impact of transient leukemia in neonates with down syndrome. Blood 111: 2991-2998, 2008.

14. Izraeli S1, Rainis L, Hertzberg L, Smooha G and Birger Y: Trisomy of chromosome 21 in leukemogenesis. Blood Cells Mol Dis 39: 156-159, 2007.

15. Roy A, Roberts I, Norton A and Vyas P: Acute megakaryoblastic leukaemia (AMKL) and transient myeloproliferative disorder (TMD) in down syndrome: a multi-step model of myeloid leukaemogenesis. Br J Haematol 147: 3-12, 2009.

16. Marosi C, Bettelheim P, Geissler K, et al: Translocation $(16 ; 21)$ (p11;q22) in acute monoblastic leukemia with erythrophagocytosis. Cancer Genet Cytogenet 54: 61-66, 1991.

17. Nobbs MC, Chan-lam D, Howell RT, Kitchen C and Copplestone JA: Acute nonlymphocytic leukemia with $\mathrm{t}(16 ; 21)$. Cancer Genet Cytogenet 70: 144-145, 1993.

18. Tan L, Tan H, Liu WD and Li HM: Clinical and laboratory investigation of hematological malignancies with $\mathrm{t}(16 ; 21)(\mathrm{p} 11 ; \mathrm{q} 22)$ translocation. Laboratory Medicine and Clinic 8: 2440-2441, 2011 (In Chinese).

19. Wu Y, Xue Y, Pan J and Ma Q: Clinical and experimental studies on five cases of acute myeloid leukemia with translocation t(16;21)(p11;q22). Zhonghua Yi Xue Yi Chuan Xue Za Zhi 20: 171-173, 2003 (In Chinese). 